\title{
Del ISET al InDRE. V. Instituto de Diagnóstico y Referencia Epidemiológicos. Posición estratégica global, 2012-2019
}

José A. Díaz-Quiñonez, ${ }^{*}$ Jorge A. Ramírez-Hernández, ${ }^{1}$ Martha E. Rodríguez-Pérez, ${ }^{2}$

Carlos Viesca-Treviño y Carmen Guzmán-Bracho ${ }^{3}$

${ }^{1}$ Universidad Nacional Autónoma de México, Facultad de Medicina, División de Estudios de Posgrado; ${ }^{2}$ Universidad Nacional Autónoma de México, Facultad de Medicina, Departamento de Historia y Filosofía de la Medicina; ${ }^{3}$ Instituto de Diagnóstico y Referencia Epidemiológicos. Ciudad de México, México

\section{Resumen}

En este documento se describen los cambios en el Instituto de Diagnóstico y Referencia Epidemiológicos (InDRE) de 2012 a 2019, las modificaciones administrativas y de equipamiento, la nueva sede y las modificaciones jurídicas al Sistema Nacional de Vigilancia Epidemiológica. Se menciona el proceso de mudanza, en especial el cuidadoso traslado del material biológico que resguarda el Instituto y se analiza la nueva forma de estudiar los brotes epidémicos, los padecimientos endémicos y la red negativa. Respecto al ámbito internacional, se describe el fomento de la vinculación con redes globales de la Organización Panamericana de la Salud, la Organización Mundial de la Salud (OMS) y otros organismos internacionales. También se menciona la designación en el InDRE de cuatro centros colaboradores de la OMS. La Red de Laboratorios de la Iniciativa Global para la Seguridad en Salud reconoció el liderazgo del InDRE, cuyo director ocupó la copresidencia del grupo de trabajo en el periodo de estudio.

PALABRAS CLAVE: Instituto de Diagnóstico y Referencia Epidemiológicos. Historia de la medicina. Salud pública.

\section{From ISET to InDRE. V. Institute of Epidemiological Diagnosis and Reference. Global strategic position, 2012-2019}

\begin{abstract}
This document describes the changes at the Institute of Epidemiological Diagnosis and Reference (InDRE) from 2012 to 2019 , the administrative and equipment modifications, the new headquarters and the National System of Epidemiological Surveillance legal modifications. The process of relocation is mentioned, especially the careful transfer of the biological material protected by the Institute, and the new way of studying epidemic outbreaks, endemic diseases and the negative network is analyzed. At the international level, the promotion of links with global networks of the Pan American Health Organization, the World Health Organization (WHO) and other international organizations is described. The designation of four WHO collaborating centres granted to InDRE is also mentioned. The Global Health Security Initiative Laboratory Network acknowledged InDRE's leadership, which co-chaired the working group during the study period.
\end{abstract}

KEY WORDS: Institute of Epidemiological Diagnosis and Reference. History of medicine. Public health.

Correspondencia:

*José A. Díaz-Quiñonez

E-mail: adiazq@unam.mx
Fecha de recepción: 20-12-2019

Fecha de aceptación: 03-01-2020

DOI: 10.24875/GMM.M20000383
Gac Med Mex. 2020;156:237-246

Disponible en PubMed

www.gacetamedicademexico.com

0016-3813/@ 2020 Academia Nacional de Medicina de México, A.C. Publicado por Permanyer. Este es un artículo open access bajo la licencia CC BY-NC-ND (http://creativecommons.org/licenses/by-nc-nd/4.0/). 
.. lo que el árbol tiene de florido, vive de lo que tiene sepultado. Francisco Luis Bernárdez

\section{Introducción}

Este artículo da continuidad al número IV de la serie del ISET al InDRE, ${ }^{1}$ en el que se describió la compleja operación de la Red Nacional de Laboratorios de Salud Pública (RNLSP).

Los cambios en el InDRE en el periodo 2012-2019 repercutieron en el conjunto del Sistema Nacional de Vigilancia Epidemiológica (SINAVE), que se organizó conforme a la Norma oficial mexicana NOM-017SSA2-2012, para la vigilancia epidemiológica (Figura 1). Por un lado, se encuentran el Comité Nacional de Vigilancia Epidemiológica, la Dirección General de Epidemiología, el InDRE y las unidades de vigilancia epidemiológica; y, por otro, los flujos de información y las muestras, cuya puerta de entrada son las unidades de vigilancia epidemiológica de todas las instituciones del sector salud. ${ }^{2}$ Todo el proceso es regulado por el Comité Nacional de Vigilancia Epidemiológica y la Dirección General de Epidemiología. Cuando el SINAVE requiere la confirmación de los casos, se incorporan la RNLSP y el InDRE. ${ }^{1}$

En 2013, el InDRE cambió de sede, aunque los preparativos habían iniciado desde el año anterior. Para la movilización escalonada de equipos y grupos de trabajo de la institución fueron necesarios tres meses. El 7 de abril de 2014 se inauguraron formalmente las nuevas instalaciones.

\section{En torno al cambio de sede institucional}

\section{Modificaciones administrativas $y$ laborales}

Como parte de la estrategia de gestión del cambio hacia la modernización y sistematización de las atribuciones del InDRE, en 2012 se implementó el Sistema de Gestión de Calidad, con un enfoque de procesos y basado en los riesgos. La incorporación del estándar de gestión de riesgo biológico para laboratorios, la norma CWA15793:2011, consolidó el modelo de gestión integral en el Instituto, único hasta hoy en el mundo para laboratorios nacionales de referencia.

La inminencia del cambio de sede institucional generó arraigo y sentido de pertenencia en el personal, al mismo tiempo que incertidumbre e inquietud. Mediante mesas de diálogo tripartitas, integradas por trabajadores, autoridades sindicales e institucionales, se acordó adecuar los perfiles de los puestos en el profesiograma, se institucionalizó laboralmente al personal que estaba bajo contrato y se permitió el cambio de adscripción de 59 trabajadores (que representaban alrededor de $10 \%$ de la plantilla). ${ }^{3} \mathrm{Al}$ mismo tiempo, se obtuvieron 67 plazas de nueva creación, que desencadenaron más de un centenar de movimientos escalafonarios.

\section{Modificaciones jurídicas}

En 2013 se actualizó la Norma oficial mexicana NOM-017-SSA2-2012, para la vigilancia epidemiológica, ${ }^{2}$ en la que el InDRE adquiere sustento legal como laboratorio nacional de referencia. El año anterior, el Instituto se había integrado a la Dirección General de Epidemiología, de acuerdo con el nuevo Reglamento Interior de la Secretaría de Salud. ${ }^{4} \mathrm{La}$ NOM-017-SSA2-2012 estableció las pautas para actualizar los criterios operativos y lineamientos técnicos del InDRE. Esos documentos han incorporado nuevos desarrollos metodológicos a los algoritmos diagnósticos y expresan la innovación tecnológica en la RNLSP. ${ }^{5}$

También en 2013, por su "alta importancia nacional", el nuevo inmueble fue designado como instalación estratégica triple A, en términos de la Ley General del Sistema Nacional de Seguridad Pública, e Instancia de Seguridad Nacional, de acuerdo con las bases de colaboración firmadas entre la Secretaría de Salud y la Secretaría de Gobernación. ${ }^{6}$

\section{Mudanza}

Durante el proceso de mudanza, el InDRE debía mantener la capacidad para efectuar los algoritmos diagnósticos "críticos" y continuar operando ante cualquier eventualidad epidemiológica que sucediera en el país. Las actividades se mantuvieron en el edificio de Santo Tomás y los integrantes de la RNLSP apoyaron mientras se trasladaban todas las fases de diagnóstico a la nueva sede. Los procesos técnicos y administrativos fueron cuidadosamente planeados y ejecutados, incluyendo la cadena de custodia y bioseguridad.

El desafío más importante de la mudanza fue la transferencia del material biológico resguardado en las instalaciones del Instituto. Constituyó un evento de seguridad nacional en términos de la gestión de riesgo biológico. 
Sistema Nacional

de Vigilancia Epidemiológica

(SINAVE)

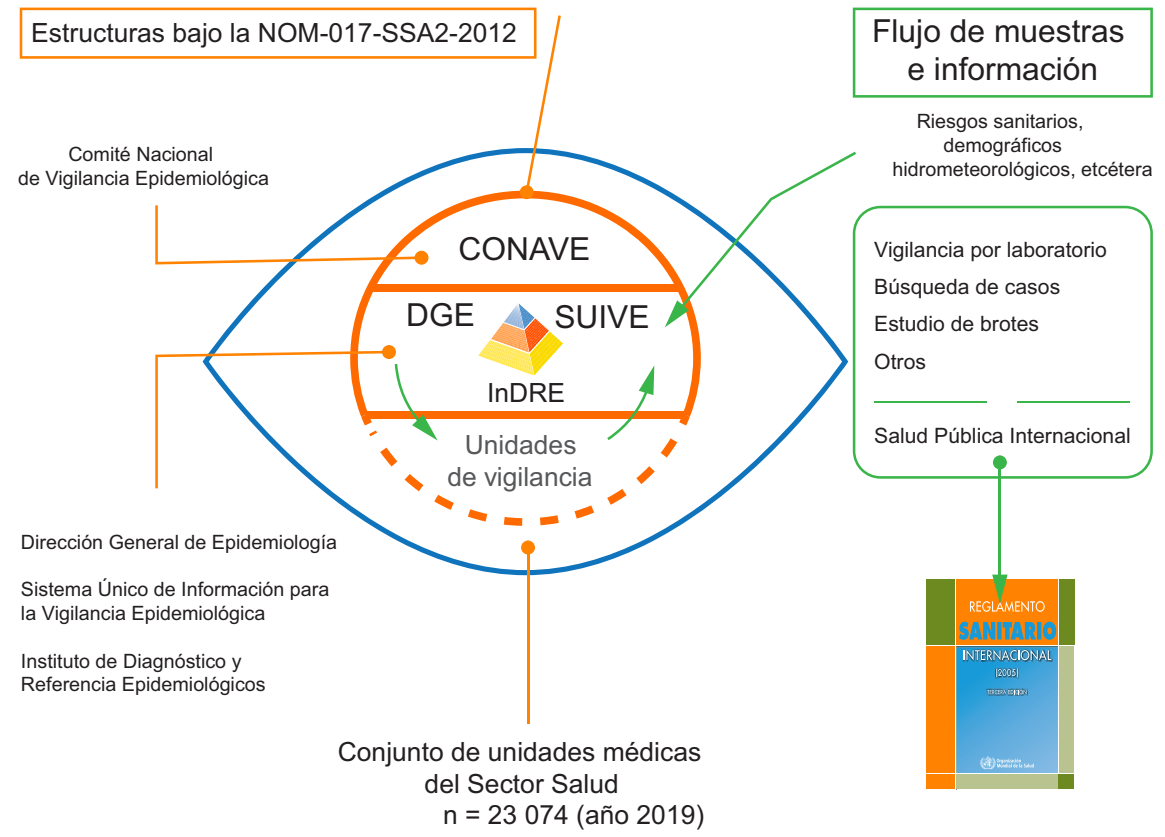

Figura 1. Esquema del Sistema Nacional de Vigilancia Epidemiológica, en el que se muestra el papel del laboratorio nacional de referencia (InDRE). México, 2019.

Después de elaborar un exhaustivo inventario del material biológico que debía ser transportado de una sede a otra, se informó a la oficina regional de la Organización Panamericana de la Salud/Organización Mundial de la Salud (OPS/OMS). Horas antes del traslado, el personal de InDRE realizó el embalaje del material, de acuerdo con los criterios internacionales. El traslado de todo el material se llevó a cabo el sábado 16 de noviembre de 2013, alrededor de la medianoche, en un solo viaje, con una caravana de camiones escoltada por elementos de la Policía Federal.

Por su relevancia, el operativo fue planeado y coordinado por funcionarios del entonces CISEN (Centro de Investigación y Seguridad Nacional ahora Centro Nacional de Inteligencia) y el InDRE, con participación del personal del gabinete legal: Secretaría de Gobernación, Secretaría de la Defensa Nacional, Secretaría de Comunicaciones y Transportes, además de la Policía Federal. El Comité Especializado de Alto Nivel en Materia de Desarme, Terrorismo y Seguridad Internacionales coordinó el cumplimiento de los acuerdos internacionales en la materia. La cédula de embarque de la OMS se firmó en las antiguas instalaciones y el proceso de movilización fue notificado a la OPS/OMS, la Oficina de Asuntos de Desarme de las Naciones Unidas y la Convención sobre Armas Biológicas.

El trabajo continuó hasta la madrugada para el personal del InDRE. Conforme se desocupaban las áreas y se desmantelaban los laboratorios, bioterios, insectarios, cuartos fríos, etcétera, se realizó la biodescontaminación y desinfección (bioseguridad ambiental) para garantizar la inocuidad de los muebles, materiales, equipos y edificios al momento de la entrega del antiguo inmueble.

Después de permanecer 78 años en la colonia Santo Tomás, el InDRE se trasladó al nuevo predio que había pertenecido a los terrenos de una antigua hacienda, luego al Manicomio General La Castañeda (1910-1968) y al Instituto Nacional de la Comunicación Humana (1968-2002). ${ }^{8}$

\section{Inauguración}

Finalmente, el 7 de abril de 2014, Día Mundial de la Salud dedicado ese año a las enfermedades transmitidas por vector, se efectuó la ceremonia oficial de inauguración de las nuevas instalaciones en presencia del presidente de la República, Enrique Peña 
Tabla 1. Red Nacional de Laboratorios de Salud Pública. Integración de las redes específicas de laboratorio. $2019^{5}$

\begin{tabular}{|c|c|c|c|c|}
\hline \multicolumn{2}{|c|}{ Red } & \multirow{2}{*}{$\begin{array}{l}\text { Laboratorios } \\
\text { estatales de salud } \\
\text { pública } \\
32\end{array}$} & \multirow{2}{*}{$\begin{array}{l}\text { Laboratorios de apoyo a la } \\
\text { vigilancia epidemiológica } \\
\qquad \text { (LAVE)* } \\
-\end{array}$} & \multirow{2}{*}{$\begin{array}{c}\text { Laboratorios } \\
\text { jurisdiccionales/ } \\
\text { locales } \\
116\end{array}$} \\
\hline 1 & Paludismo & & & \\
\hline 2 & Influenza y otros virus respiratorios & 32 & 8 & - \\
\hline 3 & Tuberculosis & 31 & - & 737 \\
\hline 4 & Dengue y otras arbovirosis & 31 & 1 & 3 \\
\hline 5 & Enfermedad diarreica aguda bacteriana & 31 & 1 & - \\
\hline 6 & Enfermedad febril exantemática & 31 & 1 & - \\
\hline 7 & Brucelosis & 31 & - & - \\
\hline 8 & Infecciones de transmisión sexual & 31 & - & - \\
\hline 9 & Hepatitis & 30 & - & - \\
\hline 10 & VIH-sida & 30 & - & - \\
\hline 11 & Enfermedad de Chagas & 29 & - & - \\
\hline 12 & Infecciones respiratorias agudas bacterianas & 28 & - & - \\
\hline 13 & Tosferina & 28 & - & - \\
\hline 14 & Leishmaniasis & 28 & - & 116 \\
\hline 15 & Rotavirus y otros enterovirus & 27 & - & - \\
\hline 16 & Entomología & 27 & - & 1 \\
\hline 17 & Rabia & 25 & - & - \\
\hline 18 & Citología del cuello uterino & 21 & - & 74 \\
\hline 19 & Leptospira & 21 & - & - \\
\hline 20 & Rickettsiosis & 4 & - & - \\
\hline
\end{tabular}

Nieto; la Secretaria de Salud, Mercedes Juan López; y la representante de la oficina de la OPS/OMS en México, Maureen Birmingham. Este acontecimiento fue comunicado en todos los periódicos de circulación nacional, en ocho primeras planas (El Universal, Reforma, Excélsior, El Sol de México, Impacto Diario, Diariolmagen, Ovaciones, Unomásuno) y en las páginas interiores del resto de la prensa escrita. La Secretaria de Salud, se refirió al InDRE de la siguiente manera: ${ }^{9}$

...principal punto de apoyo para el sistema nacional de vigilancia epidemiológica... forma parte de la estrategia de seguridad nacional para responder a las emergencias biológicas de alta peligrosidad.

En forma similar a la nota "Al fin se fundará", publicada en 1936 en El Universal con motivo de la fundación del Instituto de Salubridad y Enfermedades
Tropicales (ISET), el diario Reforma, 78 años después, tituló su nota "Inauguran, por fin, la sede del InDRE".

\section{Una nueva forma de abordar la vigilancia epidemiológica}

Actualmente, el país dispone de 20 redes de diagnóstico específico bajo la rectoría del InDRE como Laboratorio Nacional de Referencia (Tabla 1). Además de la nueva infraestructura, se sustituyó prácticamente todo el equipo de laboratorio, de informática y de comunicación. El equipo de laboratorio cambió de microscopios monoculares con lámpara incandescente a microscopios ópticos con iluminación LED y documentación digitalizada; de la bacteriología clásica, a la secuenciación genética; de las pruebas de 
aglutinación, a la quimioluminiscencia automatizada, al PCR cuantitativo en tiempo real, a la secuenciación de siguiente generación y a la bioinformática. En conjunto, estas transformaciones constituyen la renovación tecnológica más importante del Instituto desde su fundación.

Como resultado de lo anterior, hubo modificaciones en la participación del Instituto en la vigilancia epidemiológica. A continuación, se describen algunos aspectos.

\section{Brotes epidémicos}

En el periodo reseñado, el Instituto participó caracterizando los brotes epidémicos con una estrategia más rápida y de mayor valor predictivo, basada en la identificación y análisis de ácidos nucleicos por PCR en tiempo real y secuenciación de genomas completos. Un ejemplo claro de la contribución del InDRE fue la caracterización de una cepa de influenza aviar $A(H 7 N 3)$ de alta patogenicidad en trabajadores de granjas avícolas de Jalisco. ${ }^{10}$ En el Instituto también se estudió la transmisión de los virus de chikungunya ${ }^{11}, Z_{i k a},{ }^{12}$ y dengue ${ }^{13}$ en nuestro país, se identificó la circulación de enterovirus no-polio D68 en cuadros respiratorios y se descartó el diagnóstico de influenza en niños. ${ }^{14}$

La identificación del mosquito Aedes en la Ciudad de México ${ }^{15}$ detonó las actividades de monitoreo y vigilancia focalizada. ${ }^{16}$ Como consecuencia, todos los laboratorios estatales de salud pública (LESP) dispusieron, desde entonces, de metodologías moleculares para estos diagnósticos, lo que facilitó la respuesta nacional.

Durante el brote de cólera en México en el periodo 2013-2014, el diagnóstico se obtuvo con los métodos convencionales y con los métodos moleculares se evidenció la procedencia (Haití) de la cepa causante de la reemergencia de este padecimiento. Con un laboratorio móvil en campo se disminuyó el tiempo de respuesta para la atención de los individuos afectados, lo que facilitó la toma de decisiones. El brote fue controlado por el SINAVE y la participación intersectorial en tan solo 13 semanas. ${ }^{17}$

Ante la posible introducción en México de patógenos desconocidos altamente infecciosos como el Ébola y la reintroducción de otros, como el virus de la fiebre amarilla, el InDRE fortaleció la capacidad para recibirlos en su nueva sede y contenerlos en laboratorios de seguridad biológica nivel 3; también reforzó las redes de comunicación con sistemas informáticos.

\section{Endemias}

La experiencia mexicana en la erradicación del paludismo facilitó la certificación por la OMS de todos los microscopistas del Laboratorio de Paludismo del InDRE, lo cual permitió la creación del Centro Colaborador de la OMS para Malaria. Este personal certificado mantiene su alto nivel en todo el país y realiza también los diagnósticos parasitológicos de leishmaniasis y enfermedad de Chagas (comunicación personal de Juan Manuel Serna-Velázquez).

El diagnóstico serológico de la enfermedad de Chagas es indispensable para las estrategias de control de la transmisión del parásito. Para ello, el InDRE desarrolló un algoritmo de referencia nacional y un sólido programa de evaluación del desempeño en el que participan los integrantes de la RNLSP y el Centro Nacional de la Transfusión Sanguínea, para asegurar la confiabilidad diagnóstica.

La Red de Tuberculosis se inició desde la década de 1970; actualmente cuenta con 768 laboratorios de diferente complejidad en todo el país. Las pruebas bacteriológicas son la base de esta red. ${ }^{7}$

El establecimiento del programa Vigilancia Epidemiológica de las Rickettsiosis permitió la creación de la red de diagnóstico más reciente del Instituto.

En noviembre de 2019, la OMS confirió a México la primera certificación en el mundo como un país libre de rabia humana transmitida por perro, a partir de la evidencia científica que aportaron el InDRE y la RNLSP.

\section{Vigilancia de padecimientos eliminados 0 erradicados (red negativa)}

En la red negativa se incluyen enfermedades sin casos autóctonos en México² (Tabla 2). Como un ejemplo relevante, el InDRE ha participado en el proceso de erradicación de la poliomielitis, con el diagnóstico de los casos probables en los estudios de brote y mediante la vigilancia ambiental; además, es la única instancia que genera información de laboratorio para la vigilancia epidemiológica de parálisis flácida aguda en México.

\section{Reconocimientos, actividad académica y producción científica}

El Instituto recibió la acreditación ISO 15189:2012, la certificación ISO 9001:2015 y el Premio Nacional de Calidad en Salud 2015. Ese año, al InDRE se le otorgó el Premio Funsalud-GSK en la categoría de investigación epidemiológica; en 2017, un reconocimiento del 
Tabla 2. Participación del InDRE en la vigilancia de padecimientos sin registro de casos autóctonos en territorio nacional

\begin{tabular}{l|c|l|}
\hline Padecimiento & Año del último registro de casos & Procedencia \\
\hline Poliomielitis & 1990 & Jalisco \\
\hline Difteria & 1991 & Michoacán \\
\hline Sarampión* & 1996 & Distrito Federal \\
\hline Oncocercosis & 1998 & Oaxaca \\
\hline Rabia humana transmitida por perro o gato & 2004 & Sinaloa \\
\hline Ceguera por tracoma & 2007 & Chiapas \\
\hline Síndrome de rubeola congénita* & 2008 & Nuevo León \\
\hline Paludismo por Plasmodium falciparum & Sonora \\
\hline Rubeola* & 2009 & Distrito Federal y Nuevo León \\
\hline Enfermedad de Chagas transmitida por Rhodnius prolixus & 2010 & Chiapas \\
\hline Cólera* & 2012 & Sinaloa \\
\hline${ }^{* E}$ diagnóstico lo realiza toda la RnLSP. & 2018 &
\end{tabular}

Premio CANIFARMA y fue finalista en el Premio Carlos Slim a la Institución Excepcional. En 2019, resultó finalista en el Premio Nacional de Calidad de la Secretaría de Economía.

En 2014, el Consejo Nacional de Ciencia y Tecnología distinguió a la doctora Clara Gorodezky como investigadora emérita, quien además fue nombrada "El Personaje del Año 2019" en el Foro Forbes Salud, debido a sus aportes en beneficio de la salud. ${ }^{18}$

En el periodo 2012-2019 se publicaron 179 artículos en revistas indizadas con arbitraje, se depositaron 2972 secuencias genéticas y genomas completos en bases de datos públicas o restringidas y el número de miembros del Sistema Nacional de Investigadores aumentó en $143 \%$, debido a la formación e incorporación de jóvenes profesionales con posgrado.

Todos estos logros ilustran la preparación, el compromiso y el talento humano que ha desarrollado y ofrecido el InDRE al ámbito de la salud pública.

\section{Participación internacional del InDRE}

De acuerdo con la OMS, entre los 10 principales problemas de salud en 2019, seis están relacionados con enfermedades infecciosas: la influenza como un evento pandémico, la resistencia a los antimicrobianos, la amenaza de dispersión del virus del Ébola y otros patógenos (incluyendo la enfermedad $\mathrm{X}$, que representa la necesidad de prepararse para un patógeno desconocido que podría causar una grave epidemia), la renuencia a la vacunación, el dengue y el virus de la inmunodeficiencia humana.
Además de las intensas reuniones técnicas, el InDRE ha participado en muchas otras sobre políticas de laboratorios nacionales, planeación, regulación, gestión del riesgo biológico y financiamiento. También ha mantenido su colaboración con las redes globales y se ha incorporado a otras nuevas (Tabla 3 ). El Centro Nacional de Influenza del InDRE se ha mantenido desde $1951^{19}$ y fue redesignado en 2016. El Laboratorio de Tuberculosis del InDRE ha sido laboratorio supranacional para Centroamérica desde 2005; en 2016 fue acreditado como miembro de la Red de Laboratorios Supranacionales de Referencia en Tuberculosis por el Programa Mundial de Tuberculosis de la OMS. ${ }^{20,21}$

A través del Comando Norte de los Estados Unidos, en 2017 el InDRE recibió un secuenciador de siguiente generación para disminuir los tiempos de respuesta en los eventos epidémicos, como los de Zika, influenza y enfermedades transmitidas por alimentos. ${ }^{22}$

El InDRE forma parte de la Red de Laboratorios de la Iniciativa Global para la Seguridad en Salud (Tabla 3), en la que solo participan los siete países más industrializados y México; ${ }^{23}$ el director del InDRE fue elegido, por unanimidad, como copresidente para el periodo 2012-2019. México fue sede de las reuniones de ese organismo en 2014 y 2017.

\section{Centros colaboradores de la OMS en el InDRE}

Entre 2012 y 2019, el Instituto recibió designaciones importantes por parte de la OPS/OMS (Tabla 4). Un 
Tabla 3. Participación del InDRE en redes globales y multinacionales de diagnóstico

\begin{tabular}{l|l|l|l|l|l} 
Programa & Organismo coordinador
\end{tabular}

\section{Redes globales de la OPS/OMS}

The Global Polio Laboratory Network

Global Influenza Surveillance and Response System (GISRS)

WHO External Quality Assessment Project for the detection of influenza viruses

Red Mundial de Laboratorios de Sarampión y Rubéola

Sistema de Redes de Vigilancia de los Agentes Responsables de Neumonías y Meningitis Bacterianas

Red Latinoamericana de Vigilancia de la Resistencia a los Antimicrobianos

Red Panamericana de Citología

Global Salm-Surv

Red de Laboratorios para el Diagnóstico de Arbovirus

Vigilancia Genómica del Dengue en las Américas

Evaluación Externa del Desempeño de Diagnóstico de las Enfermedades Infecciosas Emergentes y Reemergentes

Programa de Control de Calidad en Bacteriología y Resistencia a los Antimicrobianos

Programa de Evaluación Externa del Desempeño OPS del Diagnóstico Microscópico de Paludismo para los Países de Mesoamérica y el Caribe

TB Supranational Reference Laboratory Network

Programa de Control de Calidad para la Susceptibilidad a Drogas de Mycobacterium tuberculosis

Red para la Evaluación de la Efectividad de la Vacuna en Latino América y el Caribe. Influenza

The External Quality Assurance System of the WHO Global Foodborne Infections Network
OMS

OMS

OMS/Centre for Health Protection, (Hong Kong)

OPS

OPS

OPS

OPS

OMS

OPS

OMS/OPS

OPS/OMS/Centro Nacional de

Enfermedades Tropicales (Bolivia)

OPS/Instituto Nacional de

Enfermedades Infecciosas (Argentina)

(a)

\section{OMS}

OPS/OMS

OPS/CDC (Estados Unidos)

OMS/Technical University of Denmark (Dinamarca)

\section{Objetivo}

Diferenciar los poliovirus de otras causas de parálisis flácida aguda

Red global para la vigilancia epidemiológica y virológica de influenza

Asegurar la calidad y mejorar la capacidad de los laboratorios a nivel mundial para la detección y subtipificación del virus de la influenza (apoyo a GISRS)

Monitorear y verificar la transmisión viral y monitorear el perfil de susceptibilidad de una población

Vigilancia epidemiológica de las neumonías y meningitis bacterianas

Vigilar la resistencia en los agentes patógenos nosocomiales y de la comunidad

Mejorar la calidad del resultado de la prueba de Papanicolaou

Fortalecer la vigilancia de enfermedades transmitidas por alimentos. Alerta y respuesta frente a brotes

Fortalecer el diagnóstico para asegurar una respuesta oportuna a brotes y epidemias

Impulsar estudios de vigilancia genómica del dengue y otros arbovirus en la Región de las Américas

Evaluar la capacidad de los laboratorios nacionales de referencia de la Región de las Américas

Programa de Control de Calidad de apoyo a ReLAVRA

Establecer el procedimiento técnico para la organización, diseño y evaluación de los laboratorios de referencia nacional de los países de la subregión

Vigilancia de la resistencia a drogas de Mycobacterium tuberculosis

Fortalecer la capacidad de los laboratorios en los países para identificar la magnitud global de la resistencia de Mycobacterium tuberculosis

Proveer información sobre la efectividad de la vacuna contra la influenza estacional

Evaluar la capacidad de los Estados miembros para detectar y responder a brotes de enfermedades transmitidas por alimentos y la aparición de resistencia a los antimicrobianos 
Tabla 3. Participación del InDRE en redes globales y multinacionales de diagnóstico (Continuación)

\begin{tabular}{|c|c|c|}
\hline Programa & Organismo coordinador & Objetivo \\
\hline \multicolumn{3}{|c|}{ Redes multinacionales } \\
\hline $\begin{array}{l}\text { Global Health Security Action Group- Laboratory } \\
\text { Network }\end{array}$ & $\begin{array}{l}\text { Global Health Security Initiative } \\
\text { (Grupo de los Siete + México) }\end{array}$ & $\begin{array}{l}\text { Asociación entre laboratorios de los países del Grupo } \\
\text { de los Siete y México, para fortalecer la preparación y } \\
\text { respuesta de salud pública global ante amenazas de } \\
\text { terrorismo químico, biológico y radiológico y nuclear }\end{array}$ \\
\hline $\begin{array}{l}\text { International Proficiency Testing Scheme for the } \\
\text { Leptospirosis MAT }\end{array}$ & $\begin{array}{l}\text { International Leptospirosis Society } \\
\text { (National Serology Reference } \\
\text { Laboratory, Australia) }\end{array}$ & $\begin{array}{l}\text { Mejorar el diagnóstico de leptospirosis en el } \\
\text { mundo }\end{array}$ \\
\hline Performance Evaluation Program & $\begin{array}{l}\text { Division of Laboratory Systems, } \\
\text { CDC }\end{array}$ & $\begin{array}{l}\text { Mejorar la vigilancia y la salud pública, la calidad } \\
\text { y seguridad del laboratorio clínico, la ciencia de } \\
\text { datos, biorrepositorios, y la competencia laboral }\end{array}$ \\
\hline PulseNet y PulseNet-NGS & $\mathrm{CDC}$ & $\begin{array}{l}\text { Comparar patrones de ADN bacteriano obtenido } \\
\text { de individuos involucrados en brotes relacionados } \\
\text { con seguridad alimentaria }\end{array}$ \\
\hline Laboratory Response Network & $\mathrm{CDC}$ & $\begin{array}{l}\text { Red de laboratorios que pueden responder a } \\
\text { amenazas biológicas y químicas, así como a otras } \\
\text { emergencias de salud pública }\end{array}$ \\
\hline Global Microbe Identifier-Proficiency Test & $\begin{array}{l}\text { Technical University of Denmark } \\
\text { (Dinamarca) }\end{array}$ & $\begin{array}{l}\text { Armonizar y estandarizar la secuenciación del } \\
\text { genoma completo de patógenos emergentes, } \\
\text { análisis de datos y bioinformática }\end{array}$ \\
\hline $\begin{array}{l}\text { United Nations Secretary-General's Mechanism- } \\
\text { Proficiency Test }\end{array}$ & $\begin{array}{l}\text { United Nations Office for } \\
\text { Disarmament Affairs/Technical } \\
\text { University of Denmark }\end{array}$ & $\begin{array}{l}\text { Fortalecer la capacidad para detectar una } \\
\text { amenaza biológica, con base en el análisis } \\
\text { genómico y bioinformático }\end{array}$ \\
\hline
\end{tabular}

Centro Colaborador de la OMS (CCOMS) es una institución designada para realizar actividades de apoyo a los programas de salud pública de la OMS. El InDRE recibió cuatro designaciones como CCOMS:

- WHO Collaborating Center for Training on Malaria Microscopy Diagnosis. ${ }^{24}$

- WHO Collaborating Center on Laboratory Biosafety. ${ }^{25}$

- WHO Collaborating Centre for Arboviruses. ${ }^{26}$

- WHO Collaborating Centre on Laboratory Quality Management. ${ }^{27}$

Con estas designaciones, en el InDRE se sitúa el $28 \%$ de los CCOMS establecidos en el país. ${ }^{28}$

\section{Discusión}

La apertura de nuevas y modernas instalaciones estratégicas, la reingeniería de procesos, la renovación tecnológica, la gestión de nuevas plazas y los movimientos escalafonarios asociados generaron un ambiente favorable para la proyección del InDRE en el periodo 2013-2019. En su conjunto constituyen la más profunda renovación del Instituto desde su fundación en 1938.
En la revisión que presentamos se consideraron tres elementos: las enseñanzas de la recuperación histórica del ISET-InDRE, los desafíos epidemiológicos de México y la prospectiva de la institución.

El análisis histórico mostró los rasgos presentes en el Instituto desde su nacimiento:

- La forma multidimensional de abordar los problemas de salud, considerando los aspectos básicos, entomológicos, clínicos, epidemiológicos y humanísticos, para apoyar las campañas y programas sanitarios prioritarios de cada época.

- La seriedad y fortaleza de la investigación científica que se realiza en el Instituto, la cual ofrece respuestas a los problemas de salud más importantes, si bien la complicada situación interna y de las fronteras del país ha planteado desafíos a la salud pública nacional, inseparable de la salud pública global.

A partir de esta significativa biografía institucional podemos concluir que el InDRE debe mantener su fuerte sentido de identidad e impulsar el continuo e intenso desarrollo científico, tecnológico y humanístico. Con ello podrá ayudar a enfrentar tanto los 
Tabla 4. Designaciones de la Organización Mundial de Salud para el InDRE, 2012 a 2019

\begin{tabular}{|c|c|c|c|}
\hline Año de designación & Nombre del CCOMS & Programa nacional que apoya & $\begin{array}{l}\text { Programa internacional que } \\
\text { apoya }\end{array}$ \\
\hline $2016^{*}$ & National Influenza Center & $\begin{array}{l}\text { Prevención y Control de las Enfermedades } \\
\text { Respiratorias e INFLUENZA }\end{array}$ & $\begin{array}{l}\text { Global Influenza Surveillance } \\
\text { and Response System }\end{array}$ \\
\hline $2016^{* *}$ & $\begin{array}{l}\text { Laboratorio Supranacional } \\
\text { para TB }\end{array}$ & Prevención y Control de la Tuberculosis & End TB Strategy \\
\hline 2017 & $\begin{array}{l}\text { WHO Collaborating Center for } \\
\text { Training on Malaria Microscopy } \\
\text { Diagnosis }\end{array}$ & Prevención y Control del Paludismo & $\begin{array}{l}\text { Global Technical Strategy for } \\
\text { Malaria 2016-2030 }\end{array}$ \\
\hline 2017 & $\begin{array}{l}\text { WHO Collaborating Center on } \\
\text { Laboratory Biosafety }\end{array}$ & $\begin{array}{l}\text { Red Nacional de Laboratorios de Salud } \\
\text { Pública }\end{array}$ & One World, One Health Agenda \\
\hline 2017 & $\begin{array}{l}\text { WHO Collaborating Centre for } \\
\text { Arboviruses }\end{array}$ & $\begin{array}{l}\text { Vigilancia Epidemiológica de Enfermedades } \\
\text { Virales Transmitidas por Vectores }\end{array}$ & $\begin{array}{l}\text { Arbovirus Diagnostic Laboratory } \\
\text { Network in the Americas }\end{array}$ \\
\hline 2018 & $\begin{array}{l}\text { WHO Collaborating Centre on } \\
\text { Laboratory Quality Management }\end{array}$ & $\begin{array}{l}\text { Red Nacional de Laboratorios de Salud } \\
\text { Pública }\end{array}$ & $\begin{array}{l}\text { Conference on Laboratory } \\
\text { Quality Systems }\end{array}$ \\
\hline $2018^{* * *}$ & Poliovirus-Essential Facilities & Vigilancia de Polio y Parálisis Flácida Aguda & $\begin{array}{l}\text { Global Polio Eradication } \\
\text { Initiative }\end{array}$ \\
\hline
\end{tabular}

padecimientos infecciosos, como los crónicos y los no transmisibles.

En diciembre de 2019, en la provincia de Wuhan, China, se inició un brote epidémico por el coronavirus SARS-CoV2, causante de Covid-19, enfermedad que rápidamente se convirtió en una pandemia. Ante este nuevo desafío global, el InDRE fue la primera institución de América Latina en realizar la confirmación de casos por métodos moleculares. La metodología sería transferida en cuestión de algunos días a la RNLSP. ${ }^{29}$

La tarea diagnóstica a cargo del InDRE debe ir más allá del laboratorio e incluir aspectos económicos, sociales y ecológicos, para explicar la complejidad detrás de las cifras de morbilidad y mortalidad. En el futuro mediato, el InDRE deberá mantener el impulso en la investigación de alto nivel y vincularse con las áreas clínicas de los institutos nacionales de salud, hospitales de referencia y alta especialidad.

A partir del aprendizaje de su gran pasado, el InDRE deberá continuar en el siglo XXI como una institución de seguridad nacional, líder en la salud pública global.

\section{Agradecimientos}

Nuestro reconocimiento a la Red Nacional de Laboratorios de Salud Pública y a la comunidad del
InDRE, que a lo largo de los años han dado prueba de su enorme profesionalismo y compromiso con la salud pública de México. José A. Díaz-Quiñonez agradece a los miembros de la Red de Laboratorios de la Iniciativa Global para la Seguridad en Salud, el apoyo brindado durante su gestión como copresidente (2012-2019). A Irma López-Martínez, Lucía Hernández-Rivas, Jean-Marc Gabastou, por las entrevistas que amablemente nos concedieron.

\section{Fuentes de financiamiento}

La presente investigación no ha recibido ninguna beca específica de agencias de los sectores público, comercial, o sin ánimo de lucro.

\section{Conflicto de intereses}

Ninguno.

\section{Responsabilidades éticas}

Protección de personas y animales. Los autores declaran que para esta investigación no se han realizado experimentos en seres humanos ni en animales.

Confidencialidad de los datos. Los autores declaran que en este artículo no aparecen datos de pacientes. 
Derecho a la privacidad y consentimiento informado. Los autores declaran que en este artículo no aparecen datos de pacientes.

\section{Bibliografía}

1. Guzmán-Bracho C, Ramírez-Hernández JA, Rodríguez-Pérez ME, Viesca-Treviño C, Díaz-Quiñonez JA. Desde el ISET al InDRE. Desde el ISET al InDRE. IV. Instituto de Diagnóstico y Referencia Epidemiológicos: Nueva orientación, 1990-2012. Gac Med Mex. 2020;155:322-327.

2. Norma Oficial Mexicana NOM-017-SSA2-2012, para la vigilancia epidemiológica. México: Diario Oficial de la Federación. 2013 Feb 19.

3. Diaz-Quiñonez JA. Informe de gestión 2012-2019. En: Acta administrativa de entrega-recepción de la Dirección General Adjunta del Instituto de Diagnóstico y Referencia Epidemiológicos. México: Secretaría de la Función Pública; 2019.

4. Decreto que reforma, adiciona y deroga diversas disposiciones del Reglamento Interior de la Secretaría de Salud. México: Diario Oficial de la Federación. 2018 Feb 07.

5. Lineamientos vigentes, Red Nacional de Laboratorios de Salud Pública. Colección de Publicaciones Técnicas del InDRE. México: Secretaría de Salud [sitio web]. [Actualizado 2016 Oct ${ }^{26}$. Disponible en: Https://www. gob.mx/salud/documentos/lineamientos-vigentes-red-nacional-de-laboratorios-de-salud-publica

6. Bases generales de colaboración que en el marco de la Ley de Seguridad Nacional celebran la Secretaría de Gobernación y la Secretaría de Salud. México: Diario Oficial de la Federación. 2013 May 05.

7. Ochoa-Carrera LA. Traslado de materiales biológicos valiosos a las nuevas instalaciones del Laboratorio Nacional de Referencia de México, Instituto de Diagnóstico y Referencia Epidemiológicos (InDRE) [Tesis de Maestría en Gestión de la Salud]. México: Universidad del Valle de México; 2014.

8. Chávez-Briseño JJH. La comunicación humana como especialidad médica. Una visión al pasado, presente y futuro. Rev Mex AMCAOF. 2012;1:41-45

9. Palabras de la Secretaria de Salud, Mercedes Juan, durante la celebración del Día Mundial de la Salud, marco en el cual se inauguró el Instituto de Diagnóstico y Referencia Epidemiológicos (InDRE). México: Secretaría de Salud [sitio web]. 2019. Disponible en: http://portal.salud. gob. $\mathrm{mx} /$ redirector?tipo $=0 \& n$ seccion $=$ Boletines \& sec cion=2014-04-07_6882.html

10. Barrera-Badillo G, Ramírez-González E, Aparicio-Antonio R, Núñez-García T, Arellano-Suarez D, Alcántara-Pérez $P$, et al. Notes from the field: Highly pathogenic avian influenza $A(H 7 N 3)$ virus infection in two poultry workers-Jalisco, Mexico. MMWR Morb Mortal Wkly Rep. 2012;61:726-727.

11. Garay-Morán C, Román-Pedroza JF, López-Martínez I, Rodríguez-Martínez JC, Ruiz-Matus C, Kuri-Morales P, et al. Clinical and epidemiological characterization of chikungunya fever in Mexico. Rev Panam Salud Publica. 2017;41:E58

12. Jiménez-Corona ME, De la Garza-Barroso AL, Rodríguez-Martínez JC, Luna-Guzmán NI, Ruiz-Matus C, Díaz-Quiñonez JA, et al. Clinical and epidemiological characterization of laboratory-confirmed autochthonous cases of Zika virus disease in Mexico. PLoS Curr. 2016;15:8

13. González-Durán E, Vázquez-Pichardo M, Torres-Flores JM, Garcés-Ayala F, Méndez-Tenorio A, Curiel-Quesada E, et al. Genotypic variability analysis of DENV-1 in Mexico reveals the presence of a novel Mexican lineage. Arch Virol. 2018;163:1643-1647.

14. Vázquez-Pérez JA, Ramírez-González JE, Moreno-Valencia Y, Hernández-Hernández VA, Romero-Espinoza JA, Castillejos-López M, et al. EV-D68 infection in children with asthma exacerbation and pneumonia in Mexico City during 2014 autumn. Influenza Other Respir Viruses. 2016;10:154-160.

15. Kuri-Morales P, Correa-Morales F, González-Acosta C, Sánchez-Tejeda G, Dávalos-Becerril E, Juárez-Franco F, et al. First report of Stegomyia aegypti (=Aedes aegypti) in Mexico City, Mexico. Med Vet Entomol. 2017;31:240-242.

16. Dávalos-Becerril E, Correa-Morales F, González-Acosta C, Santos-Luna R, Peralta-Rodríguez J, Pérez-Rentería C, et al. Urban and semiurban mosquitoes of Mexico City: A risk for endemic mosquito-borne disease transmission. PLoS One. 2019;14:E0212987.

17. Díaz-Quiñonez A, Hernández-Monroy I, Montes-Colima N, Moreno-Pérez, A, Galicia-Nicolás A, Martínez-Rojano H, et al. Outbreak of Vibrio cholerae serogroup 01, serotype Ogawa, biotype El Tor strain-La Huasteca Region, Mexico, 2013. MMWR Morb Mortal Wkly Rep. 2014;63:552-553.

18. Forbes reconoce a investigadoras por aportes en beneficio de la salud. Forbes México. 2019 Jun 18.

19. Ramírez-Hernández JA, Guzmán-Bracho C, Rodríguez-Pérez ME, Viesca-Treviño C, Díaz-Quiñonez JA. Desde el ISET al InDRE. III. Instituto de Salubridad y Enfermedades Tropicales: Crisis y renovación, 19651989. Gac Med Mex. 2019;155:641-646.

20. World Health Organization [sitio web]. WHO TB Supranational Reference Laboratory Network. Suiza: WHO; 2019. Disponible en: https://www.who. int/tb/areas-of-work/laboratory/srl-network/en/

21. Fortalecimiento de la Red de Laboratorios de Tuberculosis en la Región de las Américas. Perú: Organismo Andino de Salud-Convenio Hipólito Unanue; 2019.

22. Embajadora Roberta Jacobson dona equipo médico. Embajada y Consulados de Estados Unidos en México [sitio web]; 2017. Disponible en: https://mx.usembassy.gov/es/embajadora-roberta-jacobson-dona-equipo-medico/

23. Global Health Security Initiative [sitio web]. Canadá: GHSI; 2019. Disponible en: http://www.ghsi.ca/english/background.asp

24. World Health Organization [sitio web]. WHO Collaborating Centre for Training on Malaria Microscopy Diagnosis. Suiza: WHO; 2017. Disponible en: http://apps.who.int/whocc/Detail.aspx?cc_ref=MEX-29\&cc_ code $=$ mex

25. World Health Organization [sitio web]. WHO Collaborating Center on Laboratory Biosafety. Suiza: WHO; 2017.

26. World Health Organization [sitio web]. WHO Collaborating Centre for Arboviruses. Suiza: WHO; 2017. Disponible en: http://apps.who.int/ whocc/Detail.aspx?cc_ref=MEX-31\&cc_code $=$ mex

27. World Health Organization [sitio web]. WHO Collaborating Centre on Laboratory Quality Management. Suiza: WHO; 2018. Disponible en: http://apps.who.int/whocc/Detail.aspx?cc_ref=MEX-34\&cc_code=mex

28. World Health Organization [sitio web]. WHO Collaborating Centres. Global database Mexico. Suiza: WHO; 2019. Disponible en: http://apps.who. int/whocc/List.aspx?cc_code=MEX

29. Díaz-Quiñonez JA. Emergence of novel coronavirus SARS-CoV2 in China and the response in Mexico. Gac Med Mex. 2020;156(2):91-93. DOI:10.24875/GMM.M20000345 